\title{
Styphnolobium japonicum (L.) Schott Flower Extract Alleviates Oxidative Stress and Inflammatory Factors in the Adjuvant-Induced Arthritis Rat Model
}

\author{
Tiansheng Liu' \\ Bin $\mathrm{Su}^{2}$ \\ 'Department of Joint Surgery, Tianjin \\ Hospital, Tianjin, 3002II, People's \\ Republic of China; ${ }^{2}$ Department of \\ Research, Characteristic Medical Center \\ of PAP, Tianjin, 300162, People's Republic \\ of China
}

\begin{abstract}
Introduction: This research was to evaluate the beneficial effects of Styphnolobium japonicum (L.) Schott flower extract (SJF) on the adjuvant-induced arthritis rat model.

Methods: Arthritis was evoked by injection of complete Freund's adjuvant (CFA) in the hind paw. SJF (150 or $300 \mathrm{mg} / \mathrm{kg} /$ day) or Celecoxib $(5 \mathrm{mg} / \mathrm{kg} /$ day) were administered intragastrically from the 0th day to the 28th day. The arthritis symptoms (paw edema, arthritic scores, mechanical hyperalgesia, and thermal hyperalgesia), inflammation biomarkers (RT and CRP), related enzymes (MMP1 and MMP13), oxidative stress markers (CAT, SOD, GPx, and MDA), and inflammatory cytokines (IL- 6 , IFN- $\gamma$, TNF- $\alpha$, and IL-1 $\beta$ ) of SJFtreated CFA rats were evaluated.

Results: CFA rats exhibited severe arthritis symptoms, increased oxidative stress, and inflammatory cytokines. Interestingly, SJF treatment relieving arthritis symptoms and restored body weight gain compared with those in the CFA group. SJF treatment decreased the levels of CRP, RF, MMP1, and MMP13 in the CFA group. Besides, SJF treatment increased the activities of antioxidant enzymes and decreased the MDA content and inflammatory cytokines compared with those in the CFA group. Moreover, SJF could increase the mRNA expression of GPx-1 and CAT and inhibit the mRNA expression of IL-6 and TNF- $\alpha$ in the ankle tissue of CFA rats.
\end{abstract}

Keywords: flos sophorae immaturus, arthritis, inflammatory, oxidative stress

\section{Introduction}

Arthritis is a disease with a high incidence worldwide, including more than 100 types, of which the most common are rheumatoid arthritis, inflammatory arthritis, psoriatic arthritis, osteoarthritis, gouty arthritis, and so on. ${ }^{1}$ Rheumatoid arthritis is a chronic inflammation dysfunction and auto-immune disease, which is characterized by superfluous immune-mediated pathological aberrations, such as joint swelling, pannus formation, synovial hyperplasia, and synovitis, resulting in cartilage, joint destruction, and bone erosion. ${ }^{2}$ Rheumatoid arthritis impacts $0.32-0.36 \%$ of the population in China, ${ }^{3}$ in which female patients are about 3 times higher than male patients. In addition, arthritis patients have decreased muscle work and pain, resulting in decreasing quality of life and limiting physical activity. ${ }^{4}$ Although detailed the pathogenesis and etiology of rheumatoid arthritis are not yet fully understood. However, the previous report indicated that chronic inflammation, articular devastation, and autoantibodies development are the foundation in the etiology of rheumatoid arthritis. ${ }^{5}$ Inflammation cytokines are involved in the
Correspondence: Bin Su

Department of Research, Characteristic Medical Center of PAP, No. 220 Chenglin Road, Dongli District, Tianjin, 300I62,

People's Republic of China

Email subin008I0@I63.com 


\section{Graphical Abstract}

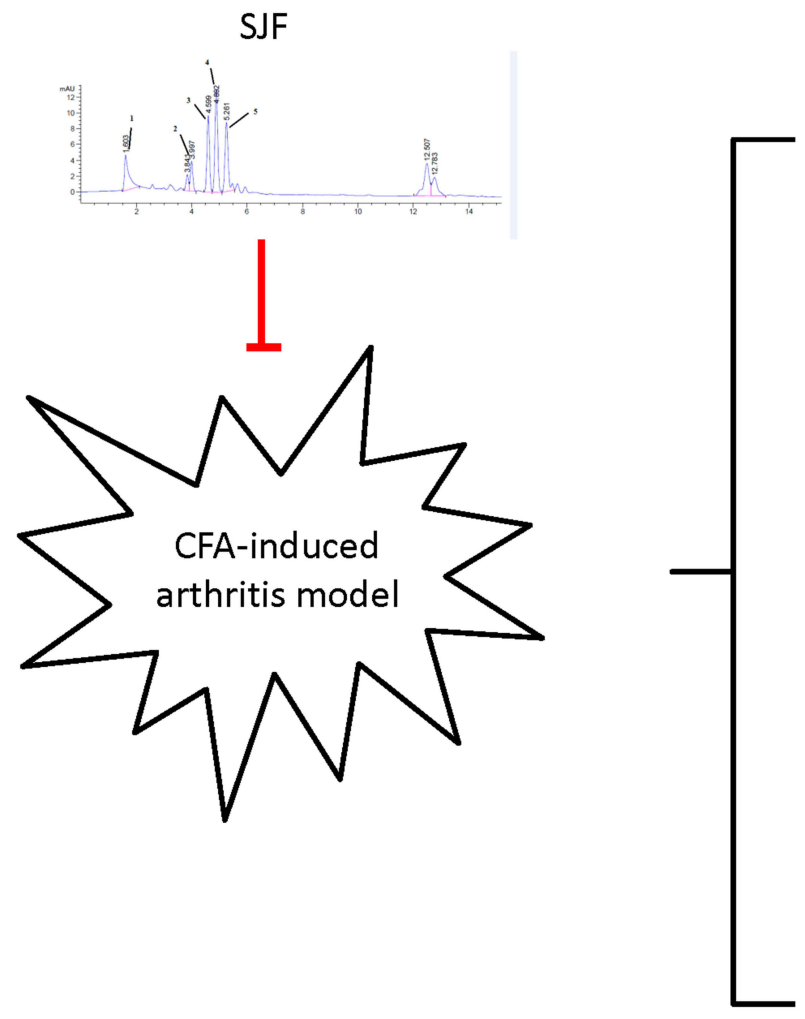

Paw edema

Arthritic scores

Pain behavioral tests

Histopathological examinations

Related enzymes: MMP1 and MMP13

Inflammation biomarkers: RF and CRP

Inflammation: TNF- $\alpha$ and IL-6

Oxidative stress: CAT and GPx-1 etiology of rheumatoid arthritis, and the unbalance between anti-inflammatory and pro-inflammatory factors determines the degree of inflammation leading to the impairment of periarticular tissue. ${ }^{6}$ Besides, the proinflammatory factors, including interleukin-6 (IL-6), IL$1 \beta$, and tumor necrosis factor- $\alpha(\mathrm{TNF}-\alpha)$ are higher in arthritis than those in normal people. The redundant of these pro-inflammatory cytokines induced the generation of chemokines, including prostaglandins, metalloproteinases, and cyclooxygenase-2, which further promotes the destruction of periarticular tissue. ${ }^{7}$ Besides the inflammation, increased oxidative stress and reactive oxygen species play an important role in the progression of periarticular tissue damage. ${ }^{8}$

Clinically, the use of disease-modifying anti-rheumatic drugs (DMARDs) and non-steroidal anti-inflammatory drugs (NSAIDs) are the main treatment strategies for arthritis disease. Although effective, the application of those drugs is limited due to serious adverse reactions, such as infections, gastrointestinal ulcers, and cardiovascular complications. ${ }^{9}$ Therefore, complementary and alternative medicines have emerged because of their efficacy and safety.
The dried flower of Styphnolobium japonicum (SJ) (L.) Schott is a Traditional Chinese Medicine and listed in the Chinese Pharmacopoeia. It has been used traditionally for the treatment of dizziness, conjunctivitis, leukorrhea, intestinal hemorrhage, hematemesis, and bleeding hemorrhoids. $^{10}$ The major components of SJ include amino acids, phospholipids, triterpene, isoflavonoids, and flavonoids. Among these components, isorhamnetin, kaempferol, genistein, quercetin, and rutin are the principal active compounds of SJ. ${ }^{11}$ These phytochemicals have been investigated to exert anti-inflammatory, antioxidant, anti-atherosclerotic, anti-osteoporotic activities. ${ }^{10}$ Besides, previous reports indicated that these flavonoids, especially genistein and kaempferol, are promising agents for arthritis treatment. ${ }^{12,13}$ Thus, we hypothesized SJ flower extract (SJF) may treat rheumatoid diseases. However, to date, it is not clear whether SJF is effective in the prevention and treatment of arthritis.

In the present research, for the first time, a rat model of arthritis was established to investigate the prevention effect of SJF against adjuvant-induced arthritis in rats. 


\section{Materials and Methods}

\section{Reagents and Materials}

Celecoxib and complete Freund adjuvant (CFA) were procured from Sigma Aldrich (St. Louis, MO, USA). Sophora Japonica L. flower was procured from Chongqing City of Guangxi Autonomous Region in China. All remaining chemicals and reagents were procured from Aladdin (Shanghai, China).

\section{Preparation and Characterization of SJF}

Dried SJ flower powder $(500 \mathrm{~g})$ was sieved through 100 mesh and extracted three times with $85 \%$ ethanol $(5 \mathrm{~L})$ by ultrasonication (the solid-liquid ratio is $1: 10$ ). Then, the combined extract was evaporated to dryness in vacuo at $60^{\circ} \mathrm{C}$. Finally, $139.7 \mathrm{~g}$ of SJF was obtained. Chromatography analysis was performed using Agilent 1260 HPLC system equipped with an Agilent ZORBAX SB C18 column $(4.6 \times 100,3.5 \mu \mathrm{m})$. The mobile phase consisted of solvents A (methanol) and B ( $0.1 \%$ formic acid in water) and the mobile phase in gradient mode as follows: 0 $\min , 5 \% \mathrm{~A} ; 18 \mathrm{~min}, 50 \% \mathrm{~A}$. The wavelength was $254 \mathrm{~nm}$. The flow rate of the mobile phase was $0.8 \mathrm{~mL} / \mathrm{min}$. And the column temperature was maintained at $35^{\circ} \mathrm{C}$.

\section{Animals}

The 6-8 weeks old Wistar rats, weighing 170-190 g were used in the present study. And all rats were kept in a stable experimental environment (relative humidity $40-60 \%$ and temperature of $20-22^{\circ} \mathrm{C}$ with $12 / 12 \mathrm{~h}$ light-dark schedules). All animals were allowed free access to a standard chow diet and water. The animal experimental protocol was approved by the Animal Ethics Committee of Tianjin Hospital (Approval number: 2020126) and research was conducted in accordance with the WHO guidelines for animal care.

\section{Animal Model Generation and SJF Administration}

After initial acclimatization for seven days, the CFA-induced arthritis model was established on day 1 by a single injection of $0.1 \mathrm{~mL}$ of CFA in the left hind rat paw. ${ }^{14}$ Animals were randomly divided into five groups ( $\mathrm{n}=8$ in each group) as follows: Control group (Control): normal rats which received $0.1 \%$ DMSO oral vehicle; CFA group (CFA): RA rats which received $0.1 \%$ DMSO oral vehicle; the SJF lowdose group (LSJF): RA rats which received SJF $(150 \mathrm{mg} / \mathrm{kg} /$ d); the SJF high-dose group (HSJF): RA rats which received SJF (300 mg/kg/d); celecoxib-treated group (Cel): RA rats which received celecoxib $(5 \mathrm{mg} / \mathrm{kg} /$ day). The selection of Cel dose was based on the previous studies. ${ }^{15,16}$ Drugs were administered intragastrically once a day for four weeks starting $1 \mathrm{~h}$ after CFA inoculation. The dose of SJF was based on the previous report and our preliminary experiment. ${ }^{17}$ The schematic representation of the experimental protocol is presented in Figure 1A.

\section{Measurement of Paw Edema and Arthritic Scores}

The paw edema of each rat was assessed using an MK-101P Plethysmometer (Muromachi) on days 0, 7, 14, 21, and 28. Besides, the arthritis scores of each rat were measured to assess the arthritis severity. The scoring criteria were showed as follows: $0=$ no swelling or erythema; $1=$ slight swelling or erythema of the digits; $2=$ moderate swelling or erythema of the digits; 3 = severe swelling or erythema of the digits; 4 $=$ ankylosis, incapacity to bend the digits.

\section{Pain Behavioral Tests}

The IITC Digital Paw Pressure Randall Selitto Meter (IITC, CA, USA) was used to evaluate mechanical hyperalgesia. Briefly, apply pressure to the plantar area of the
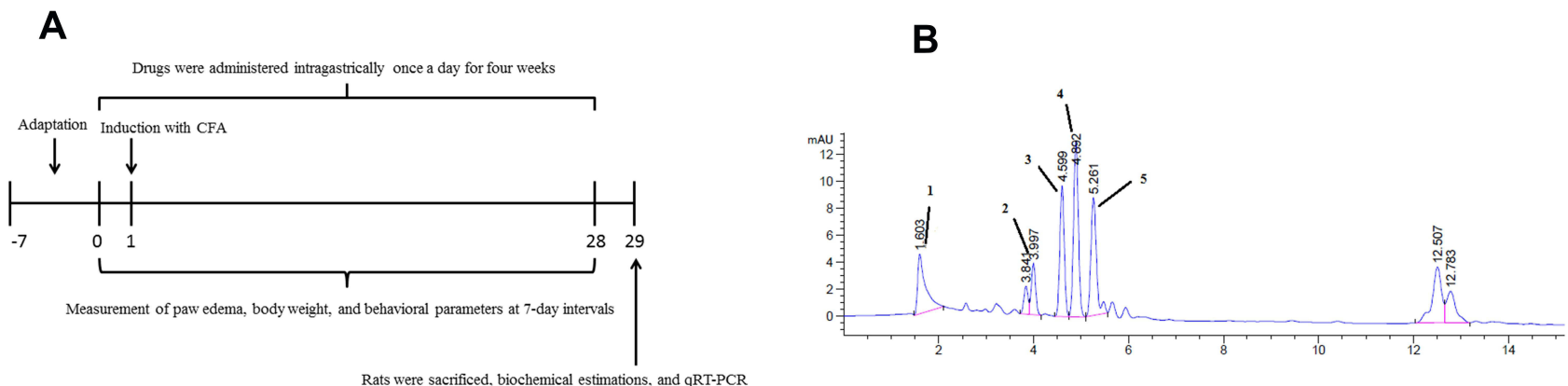

Figure I The experimental protocol of SJF on CFA rats (A). HPLC chromatogram of major ingredients in SJF (B). (I) rutin, (2) quercetin, (3) genistein, (4) kaempferol, and (5) isorhamnetin. 
hind paw at a rate of $10 \mathrm{~g} / \mathrm{s}$. A cut-off threshold of 250 $\mathrm{g}$ was performed to prevent tissue injury. The paw withdrawal threshold was defined as the minimum force required to induce a paw withdrawal response. The experiment was repeated three times for each rat, and the threshold value was averaged. ${ }^{18}$

Thermal hyperalgesia was performed by placing rats on a preheated hot plate $\left(52 \pm 1^{\circ} \mathrm{C}\right)$ based on the previous report. ${ }^{18}$ The latency reaction times of the claw, namely the flinching, jumping, and/or licking of the claw, were defined as signs of hyperalgesia. A cut-off time of 20 $\mathrm{s}$ was conducted to avoid tissue harm.

\section{Blood Collection and Ankle Joint Tissue Preparation}

At the end of the experiment, rats were anesthetized with a combination of ketamine/xylazine ( $80: 10 \mathrm{mg} / \mathrm{kg}$, ip), and then whole blood was obtained from the abdominal aorta of rats using a heparinized syringe. Subsequently, blood samples were centrifuged at $3000 \times \mathrm{g}$ for $20 \mathrm{~min}$ at $3^{\circ} \mathrm{C}$, and then the supernatant was collected and stored at $-80^{\circ} \mathrm{C}$ for future analysis.

After the whole blood collection, the ankle joint tissues were immediately removed. Subsequently, the joint tissues were homogenized in saline using a homogenizer (GlasCol, USA), and the homogenate was centrifuged at $3000 \times$ $\mathrm{g}$ for $20 \mathrm{~min}$ at $3^{\circ} \mathrm{C}$. The supernatant was collected and used for the measurement of the biochemical parameters.

\section{Histological Analysis}

The ankle joint tissues were fixed in formaldehyde (4\%) overnight. After the samples were dehydrated in a serial dilution of alcohol. The specimens were processed for paraffin embedding. Then, 5- $\mu$ m-thick tissue sections were prepared using a microtome and stained by hematoxylin-eosin (H\&E) for the light microscope examination.

\section{Determination of Rheumatoid}

\section{Biomarkers}

Matrix metalloproteinase-1 (MMP1) and MMP-13 in plasma and joint homogenates were analyzed using commercial kits on a microplate reader (Thermo Fisher, USA) according to the manufacturer's protocol. The catalog number of the corresponding kits was as follows: MMP1 (H146-5), and MMP-13 (H146-4).

\section{Determination of Inflammatory}

\section{Biomarkers and $\mathrm{PGE}_{2}$ Level in the Ankle Joints}

The levels of CRP and RF in rat serum were measured using commercially available kits (Nanjing Jiancheng Bioengineering Institute, Nanjing, China). The levels of inflammatory biomarkers (IL-6, IL-10, IFN- $\gamma$, TNF- $\alpha$, and IL-1 $\beta$ ) and $\mathrm{PGE}_{2}$ in the ankle joints were measured using commercially available kits (Nanjing Jiancheng Bioengineering Institute, Nanjing, China) on a microplate reader (Thermo Fisher, USA) according to manufacturer's protocol. The protein levels were measured using a bicinchoninic acid protein assay kit (Nanjing Jiancheng Bioengineering Institute, Nanjing, China). Inflammatory factor concentrations were showed as $\mathrm{pg} / \mathrm{mL}$ of protein. The catalog number of the corresponding kits was as follows: CRP (H126), RF (E035-1-1), PGE 2 (H099-1), IL-10 (H009), IFN- $\gamma \quad(\mathrm{H} 025), \quad$ TNF- $\alpha$ (H052), IL-6 (H007), and IL-1 $\beta$ (H002).

\section{Determination of Oxidative Stress}

\section{Biomarkers in the Ankle Joints}

The levels of oxidative stress biomarkers (Catalase, SOD, GPx, and MDA) in the ankle joints were measured using commercially available cytokine kits (Nanjing Jiancheng Bioengineering Institute, Nanjing, China) on a microplate reader (Thermo Fisher, USA) based on manufacturer's protocol. Inflammatory factor concentrations were showed as $\mathrm{pg} / \mathrm{mL}$ of protein. The catalog number of the corresponding kits was as follows: SOD (A001-3-2), CAT (A007-1-1), GPx (A005-1-2), and MDA (A003-1-2).

\section{Quantitative RT-PCR for Gene Expression}

Total RNA was isolated from ankle joint tissues using the RNeasy Mini Kit (Qiagen, USA) according to the manufacturer's protocol. The RNA was reverse-transcribed into cDNA using the GoScript Reverse Transcription kit (Thermo, USA) according to the manufacturer's protocol. The mRNA levels were quantified by RT-qPCR using an SYBR Green qPCR Master Mix (Takara, Dalian, China) for 40 cycles on the StepOnePlus RT-PCR system (Applied Biosystems). The primer sequences used to amplify mRNA were shown in Table 1. The levels of mRNA expression were normalized to the reference gene (GAPDH). 
Table I Sequences of Primers Used in This Research

\begin{tabular}{|l|l|l|}
\hline Gene & Forward Primer & Reverse Primer \\
\hline CAT & 5'-CCTGCAACGTTCTGTAAGGC-3' & 5'-ATATCAGGTTTCTGCGCGGC-3' \\
GPx-I & 5'-CTCATGACCGACCCCAAGTT-3' & 5'-GTCAGAAAGCGACGGCTGTA-3' \\
IL-6 & 5'-TGTATGAACAACGATGATGCAC-3' & 5'-CTGGCTTTGTCTTTCTTGTT-3' \\
TNF- $\alpha$ & 5'-CTTCTGTCTACTGAACTTCGGG-3' & 5'-CAGGCTTGTCACTCGAATTTTG-3' \\
GAPDH & 5'-TCGGAGTGAACGGATTTGGC-3' & 5'-TGACAAGCTTCCCGTTCTCC-3' \\
\hline
\end{tabular}

\section{The Data Statistical Analysis}

All statistical analyses were carried out using GraphPad Prism software (GraphPad Software, Inc., La Jolla, USA). Results were presented as means \pm SEM. The data from paw edema, body weight, and behavioral tests at different time points were analyzed by two-way analysis of variance (ANOVA) followed by the Bonferroni test. Other data were analyzed by one-way ANOVA, followed by Tukey's multiple comparison test; $P<0.01$ and $P<0.05$ were usually considered statistically significant.

\section{Results}

\section{Analysis of SJF by HPLC}

To explore representative anti-arthritis active ingredients of SJF, the HPLC analysis was performed. The extraction yield of SJF is $27.94 \%$. As shown in Figure 1B, HPLCUV analysis showed the major ingredients in SJF. The five flavonoids compounds were identified as (1) rutin, (2) quercetin, (3) genistein, (4) kaempferol, and (5) isorhamnetin, respectively, via the HPLC method by comparison with an authentic standard. The relative area of these flavonoids is presented in Table 2.

\section{Effects of SJF Treatment on Arthritic Symptoms in a Rat Model of Arthritis}

The paw edema and arthritic scores of CFA rats were evaluated to investigate the antiarthritis effect of SJF. In the present study, a rat model of arthritis was established by the intradermal administration of $0.1 \mathrm{~mL}$ of CFA. As shown

Table 2 The Main Ingredients of Sophora japonica L. Flower Extract (SJF). Data are Expressed as Means \pm SD $(n=3)$

\begin{tabular}{|l|c|c|c|}
\hline No. & Rt (Min) & Identification & Relative Area (\%) \\
\hline 1 & 1.60 & Rutin & 12.87 \\
2 & 3.99 & Quercetin & 5.88 \\
3 & 4.59 & Genistein & 15.61 \\
4 & 4.89 & Kaempferol & 22.59 \\
5 & 5.26 & Isorhamnetin & 17.85 \\
\hline
\end{tabular}

in Figure 2A, the injection of CFA induced a gradual increase in paw edema on day 7 and was sustained until the end of the experiment. However, treatment with SJF $(150$ or $300 \mathrm{mg} / \mathrm{kg}$ ) or Cel afforded a significant decrease of the paw edema on day 28, as compared with the CFA group $(P<0.01, P<0.05)$. Besides, administration of SJF $(150$ or $300 \mathrm{mg} / \mathrm{kg})$ or Cel resulted in a significant decline in arthritic scores on day 28 (Figure 2B).

As shown in Figure 2C, the body weight of rats in the CFA group was lower compared with the control group $(P<0.01)$. However, treatment with SJF $(150$ or $300 \mathrm{mg} /$ $\mathrm{kg}$ ) or Cel inhibited the decrease of body weight induced by CFA $(P<0.01, P<0.05)$.

\section{Effects of SJF Treatment on Mechanical} Hyperalgesia and Thermal Hyperalgesia in

\section{a Rat Model of Arthritis}

A significant decrease in mechanical hyperalgesia and thermal hyperalgesia was observed in the CFA group as compared with the control group on day 7 , which continued till day 28 (Figure $2 \mathrm{C}$ and $\mathrm{D}$ ). However, treatment with SJF (150 or $300 \mathrm{mg} / \mathrm{kg}$ ) or Cel inhibited the decrease of the mechanical hyperalgesia and thermal hyperalgesia evoked by CFA $(P<0.01, P<0.05)$ on day 28 .

\section{Effects of SJF Treatment on Inflammation Biomarkers in Rat Serum}

As shown in Figure $3 \mathrm{~A}$ and $\mathrm{B}$, there was an obvious increase in CRP and RF levels were observed in the CFA group when compared with the control group. However, treatment with SJF (150 or $300 \mathrm{mg} / \mathrm{kg}$ ) or Cel reversed these changes by decreasing CRP and RF levels.

\section{Effects of SJF Treatment on Histopathological Changes in a Rat Model of Arthritis}

The anti-arthritic effect of SJF was further confirmed by histopathological assessment (Figure 3C). Histological 


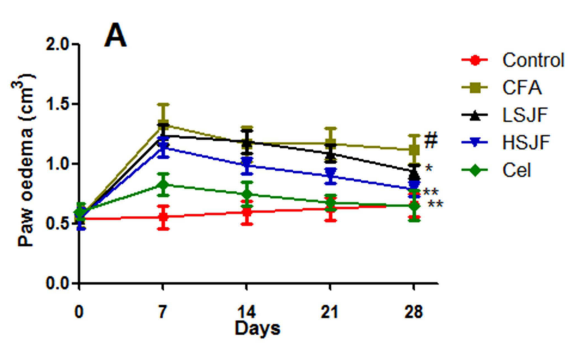

D

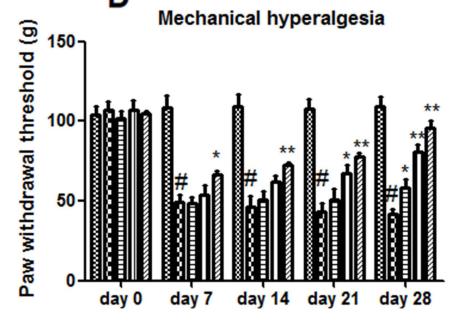

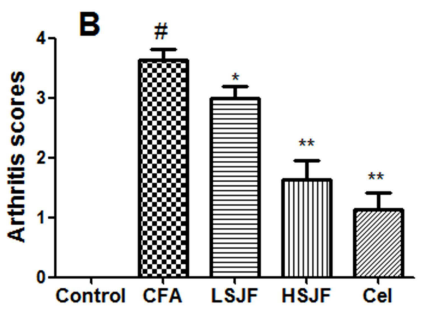

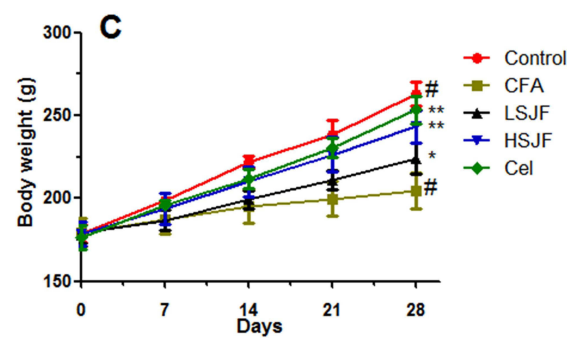

$\mathbf{E}$

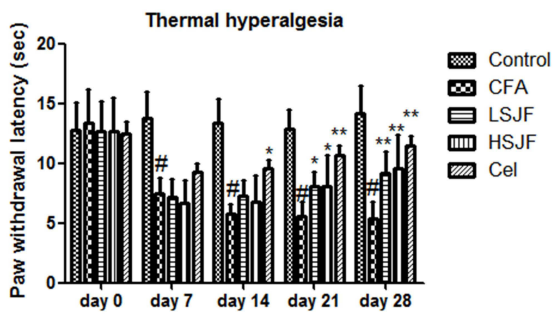

Figure 2 SJF relieved arthritis symptoms in Freund's Complete Adjuvant (CFA)-evoked arthritis rats. The paw edema (A), arthritic scores (B), body weight (C), mechanical hyperalgesia (D), and thermal hyperalgesia (E) in CFA rats after gavage administration vehicle, or SJF (I50 or $300 \mathrm{mg} / \mathrm{kg} /$ day) or Celecoxib (5 mg/kg/day) from day 0 to day 28 . Differences from paw edema, body weight, and behavioral tests were analyzed using a two-way analysis of variance (ANOVA) by Bonferroni test. Data from arthritis scores were analyzed using one-way ANOVA by Tukey's multiple comparison test. ${ }^{\#} P<0.01$ vs the control group, ${ }^{* * P}<0.01$ and $* P<0.05$ vs the CFA group. All results are shown as mean $\pm S E M$ ( $n=8$ ).

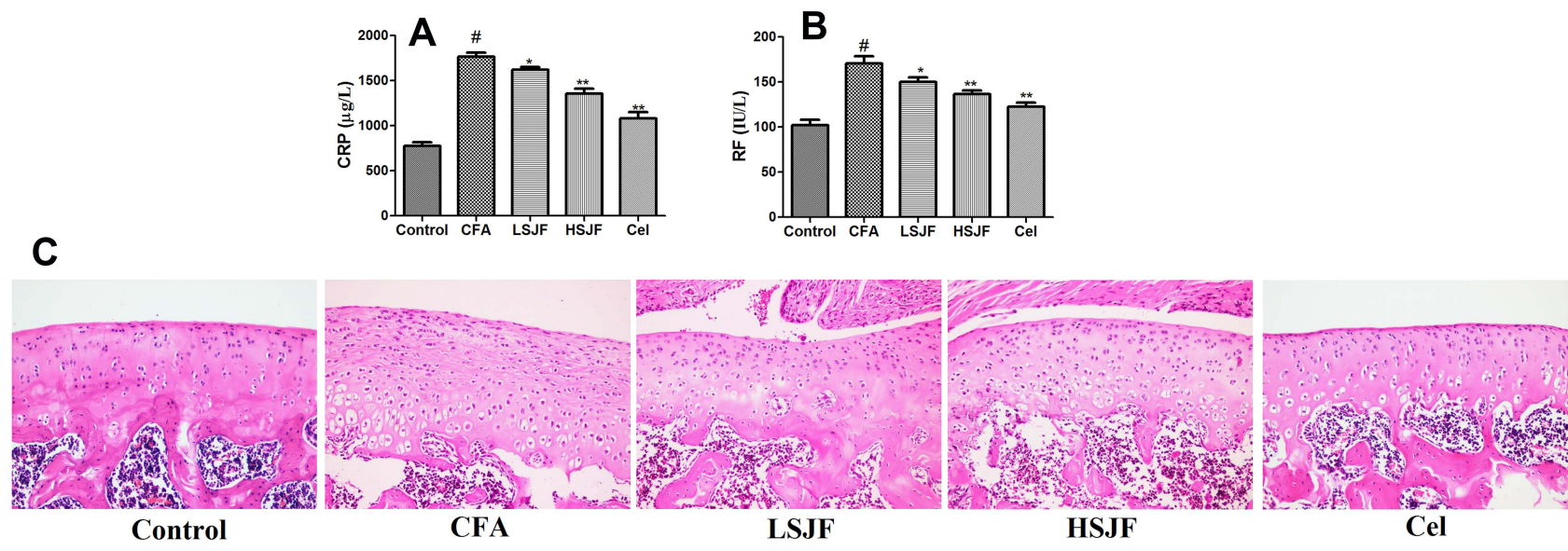

Figure 3 Effects of SJF on inflammation biomarker levels and histopathological changes in Freund's Complete Adjuvant (CFA)-evoked arthritis rats. The serum levels of CRP (A) and $\operatorname{RF}(\mathbf{B})$ were measured by ELISA. Representative photomicrographs of ankle joint tissues and stained with $\mathrm{H} \& \mathrm{E}$ (magnification $\times$ I00) $(\mathbf{C})$. Differences were analyzed using oneway ANOVA by Tukey's multiple comparison test. ${ }^{\#} p<0.01$ vs the control group, ${ }^{* * P}<0.01$ and $* P<0.05$ vs the CFA group. All results are shown as mean \pm SEM ( $=8$ ).

results indicated that ankle joint tissues of the control group showed normal morphology and appearance. Cartilage erosion, synovial hyperplasia, and inflammatory cell infiltration were observed in ankle joint tissues of the CFA group. Treatment with SJF, especially HSJF and Cel, obviously improved the CFA-induced histopathological alterations.

\section{Effects of SJF Treatment on MMPs Levels in a Rat Model of Arthritis}

Because the increased expressions of MMPs were involved in the etiopathogenesis of arthritis. Thus, the effects of SJF on
MMPs levels were also investigated. A significant increase of MMP-1 and MMP-3 was observed in the CFA group when compared with the control group (Figure 4). However, treatment with SJF (150 or $300 \mathrm{mg} / \mathrm{kg}$ ) or Cel reversed these changes by decreasing the MMP-1 and MMP-3 levels (both in plasma and joint homogenates) $(P<0.01, P<0.05)$.

\section{Effects of SJF Treatment on Inflammatory and Cytokine Mediators in Rat Ankle Joints} It has been reported that inflammatory cytokines play an important role in the etiopathogenesis of arthritis. 

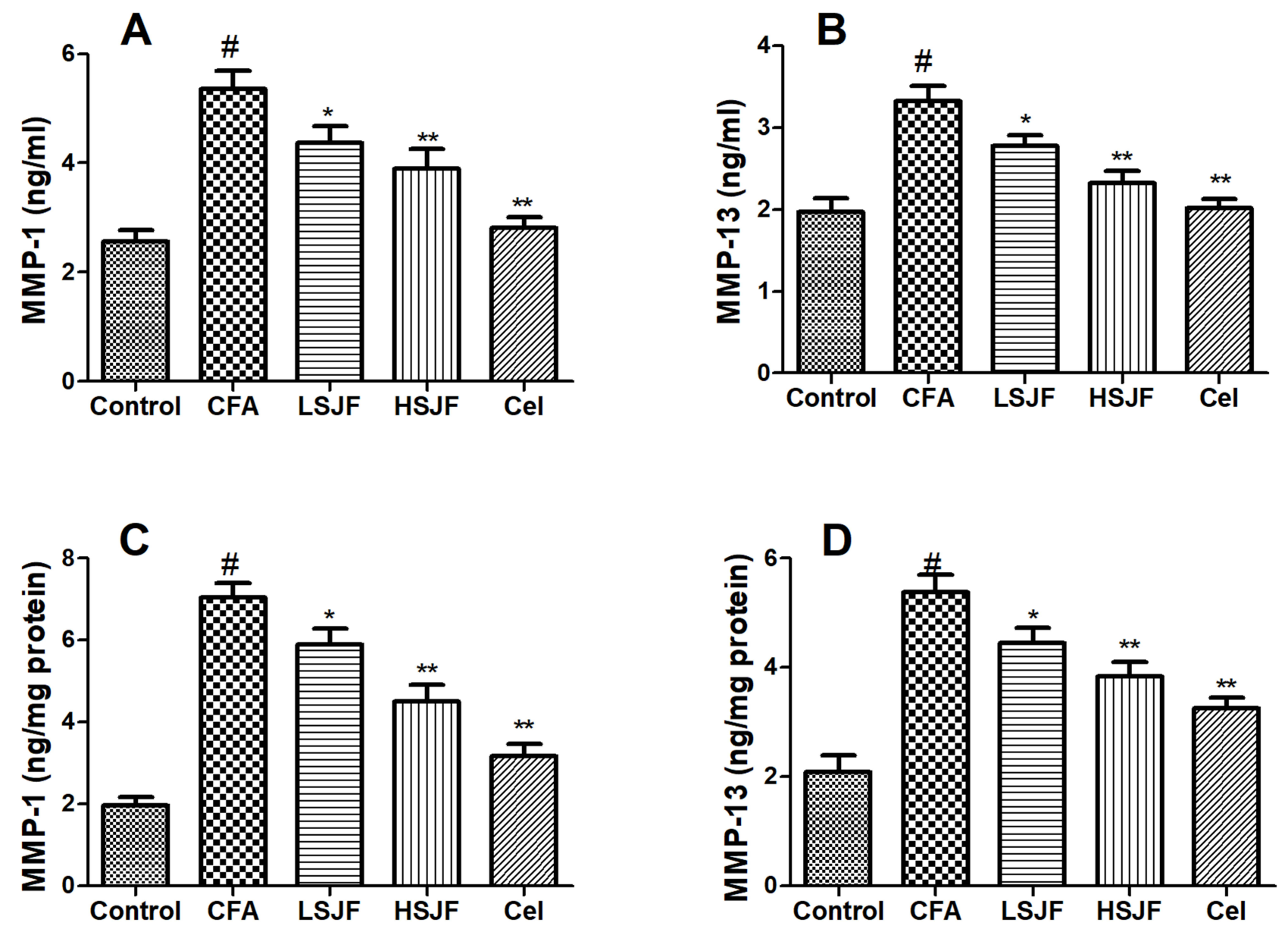

Figure 4 Effects of SJF on MMPs levels in Freund's Complete Adjuvant (CFA)-evoked arthritis rats. The plasma levels of MMP-I (A) and MMP-I3 (B) were measured by ELISA. MMP-I (C) and MMP-I3 (D) levels in joint homogenates. Differences were analyzed using one-way ANOVA by Tukey's multiple comparison test. ${ }^{\#} P<0.01$ vs the control group, $* * P<0.01$ and $* P<0.05$ vs the CFA group. All results are shown as mean \pm SEM $(n=8)$.

Therefore, the levels of inflammatory cytokines in joint homogenates were measured by ELISA. Consistent with the results of paw edema and arthritis scores, as shown in Figure 5, there was an obvious increase in IL-6, $\mathrm{PGE}_{2}$, IFN- $\gamma$, TNF- $\alpha$, and IL- $1 \beta$ levels were observed in the CFA group when compared with the control group, while an obvious decrease in IL-10 level was observed in CFA group when compared with the control group $(P<0.01)$. However, treatment with SJF (150 or $300 \mathrm{mg} / \mathrm{kg}$ ) or Cel reversed these changes by decreasing IL-6, PGE2, IFN- $\gamma$, TNF- $\alpha$, and IL-1 $\beta$ levels, and increasing IL-10 level $(P<$ 0.01 ), indicating that the antiarthritis effects of SJF might be mediated via the inhibition of inflammation.

\section{Effects of SJF Treatment on Oxidative} Stress Biomarkers in Rat Ankle Joints

It has been demonstrated that excessive oxidative stress was implicated in the development of arthritis. Therefore, the CAT, SOD, GPx activities, and MDA levels were measured to explore the effect of SJF on oxidative stress in CFA rats. As shown in Figure 6, there was an obvious decrease in catalase (CAT), SOD, and GPx levels were observed in the CFA group when compared with the control group, while an obvious increase in MDA content was observed in the CFA group when compared with the control group $(P<0.01)$. However, treatment with SJF (150 or $300 \mathrm{mg} / \mathrm{kg}$ ) or Cel reversed these changes by improving the catalase, SOD, and GPx activities, and decreasing MDA content $(P<0.01)$.

\section{Effects of SJF Treatment on the Gene Expression of Oxidative Stress Markers and Pro-Inflammatory Cytokines in Rat Ankle Joints}

To further investigate the anti-inflammatory and antioxidant actions of SJF in CFA rats, the mRNA expression of 

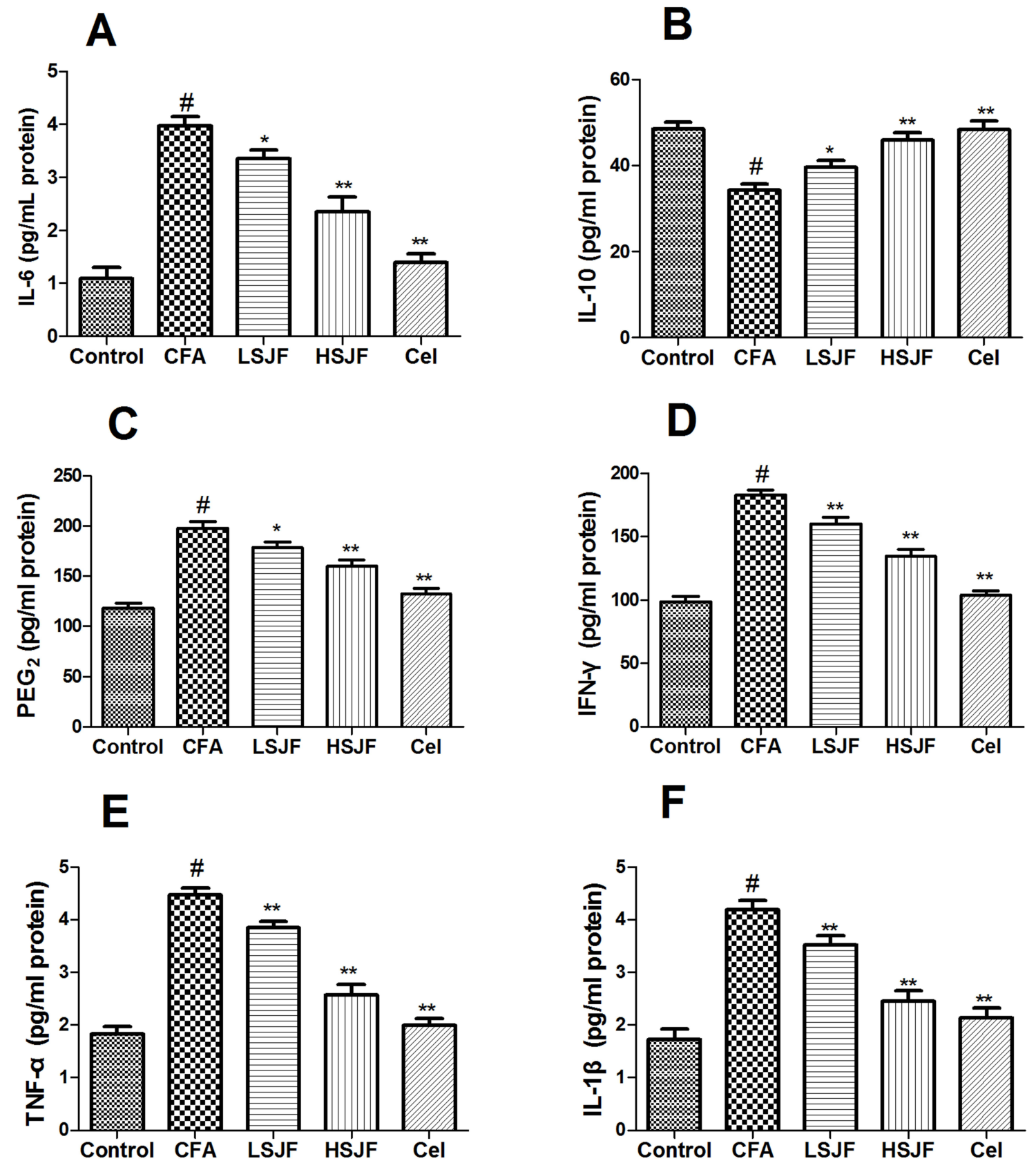

Figure 5 Effects of SJF on inflammatory and cytokine mediators in rat ankle joints. The levels of IL-6 (A), IL-I0 (B), PGE 2 (C), IFN- $\gamma(\mathbf{D})$, TNF- $\alpha$ (E), and IL-I $\beta$ (F) in joint homogenates were measured by ELISA. Differences were analyzed using one-way ANOVA by Tukey's multiple comparison test. ${ }^{*} P<0.0 \mathrm{I}$ vs the control group, ${ }^{* *} P<0.0 \mathrm{I}$ and ${ }^{*} P<0.05$ vs the CFA group. All results are shown as mean $\pm \operatorname{SEM}(n=8)$.

CAT, GPx-1, IL-6, and TNF- $\alpha$ genes was measured by qRT-PCR. As shown in Figure 7, there was an obvious decrease in the mRNA expression levels of CAT and GPX1 were observed in the CFA group when compared with the control group, while an obvious increase in IL-6 and TNF- $\alpha$ mRNA expression levels were observed in the CFA group when compared with the control group $(P<0.01)$. However, treatment with SJF (150 or $300 \mathrm{mg} / \mathrm{kg}$ ) or Cel reversed these changes by up-regulating CAT and GPx-1 expression levels, and down-regulating IL-6 and TNF- $\alpha$ mRNA expression levels $(P<0.01, P<0.05)$. Thus, the underlying mechanisms of SJF on anti-inflammatory and anti-oxidant was to down-regulate pro-inflammatory cytokines mRNA expressions and up-regulate antioxidant enzyme mRNA expressions, and then alleviate the progression of arthritis. 

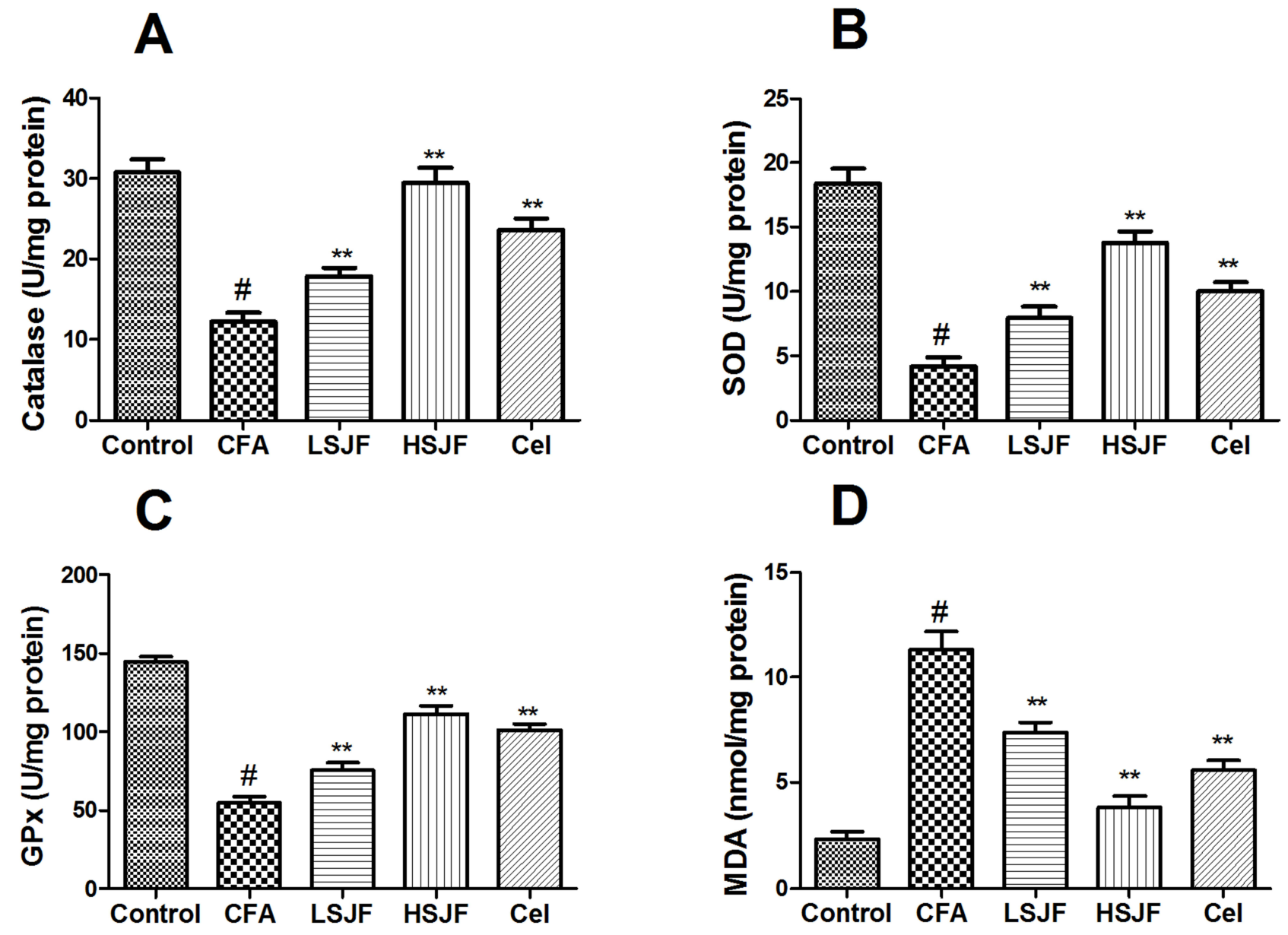

Figure 6 Effects of SJF on oxidative stress markers in rat ankle joints. The levels of Catalase (A), SOD (B), GPx (C), and MDA (D) in joint homogenates were measured by ELISA. Differences were analyzed using one-way ANOVA by Tukey's multiple comparison test. ${ }^{\#} P<0.01$ vs the control group, ${ }^{* * P}<0.01$ and $* P<0.05$ vs the CFA group. All results are shown as mean $\pm \operatorname{SEM}(n=8)$.

\section{Discussion}

It is well known that CFA-evoked arthritis is similar to human arthritis clinical characteristics such as periarticular tissue, inflammation response, and oxidative stress. ${ }^{19}$ In the present study, the intradermal administration of CFA resulted in paw edema, significant decreases in the nociceptive threshold, and high arthritis scores, indicating the rat's arthritis was successfully established. Besides, we were first to demonstrate that SJF could relieve arthritis symptoms via inhibiting the inflammation response and oxidative stress in CFA-induced arthritis rats. Moreover, the major active ingredients of SJF, including rutin, quercetin, genistein, kaempferol, and isorhamnetin, were characterized by HPLC assay, and the antiarthritis activity of SJF may be partly attributed to these flavonoid constituents. In accordance with our results, the previous reports have demonstrated that administration of rutin or kaempferol exerted the protective effect against arthritis. ${ }^{20,21}$
Besides, quercetin was reported to exert a therapeutic effect in collagen-induced arthritis. ${ }^{22}$ Genistein has been reported to prevent angiogenesis developed during rheumatoid arthritis via JAK2/STAT3 signaling pathway. ${ }^{23}$ Isorhamnetin was showed to improve inflammation and alleviate articular cartilage damage in osteoarthritis rats. ${ }^{24}$ Therefore, rutin, quercetin, genistein, kaempferol, and isorhamnetin may be responsible for the primary active ingredients in SJF against arthritis.

It has been reported that arthritis may result in chronic inflammatory pain. ${ }^{25}$ And CFA generated chronic inflammatory pain attributed to trigger inflammatory cytokines that caused sensitization of the nocicurious neurons, resulting in a decrease in pain threshold. ${ }^{26}$ In our experiment, the CFA-inoculated rats presented a significant decline in mechanical hyperalgesia and thermal hyperalgesia. Our results indicated that SJF not only decreased the paw edema and arthritic scores but also restored the threshold 
A
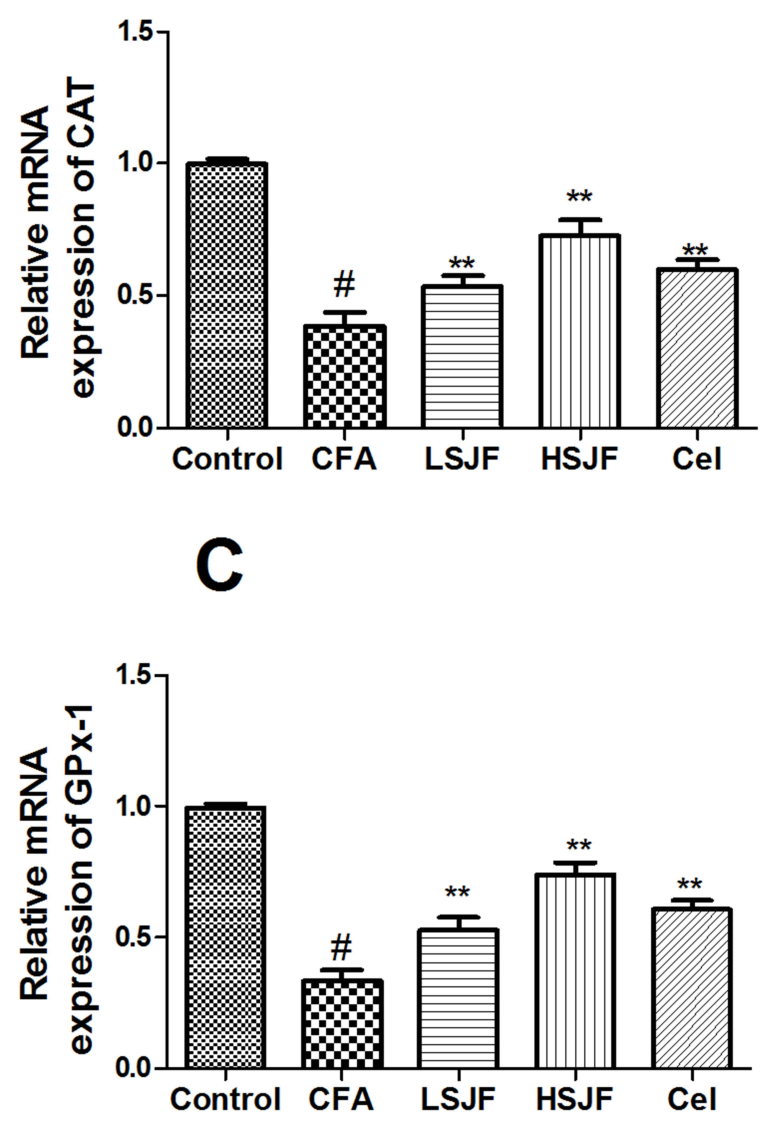

B

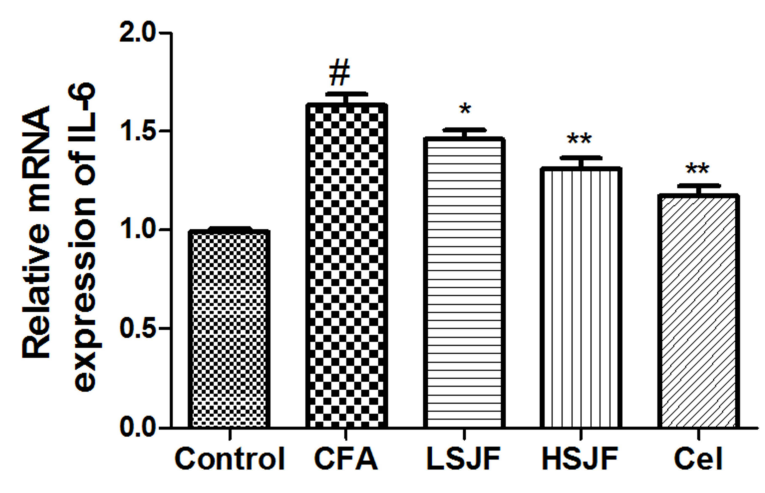

D

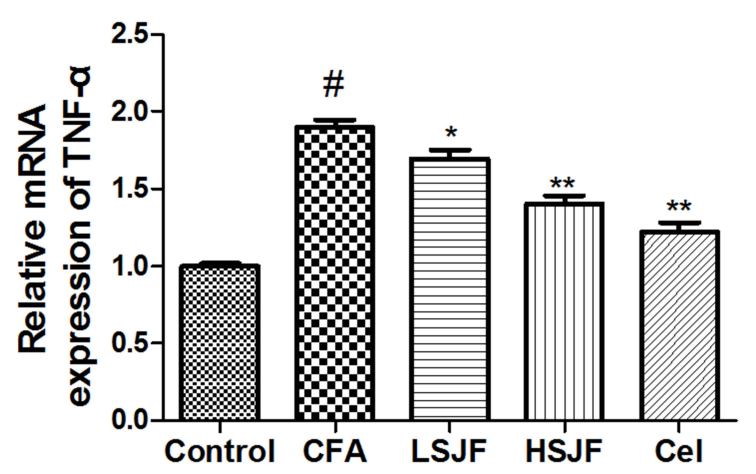

Figure 7 Effects of SJF on the gene expression of oxidative stress markers and pro-inflammatory cytokines in rat ankle joints. Relative mRNA expression of (A) CAT, (B) IL6, (C) GPx-I, and (D) TNF- $\alpha$ in the ankle joint tissue were measured by qRT-PCR. Differences were analyzed using one-way ANOVA by Tukey's multiple comparison test. ${ }^{\#} P<0.0$ l vs the control group, ${ }^{* *} P<0.01$ and ${ }^{*} P<0.05$ vs the CFA group. All results are shown as mean \pm SEM $(n=8)$.

of mechanical hyperalgesia and thermal hyperalgesia. These beneficial effects of SJF may be related to the inhibition of CFA-evoked secretion of inflammatory cytokines. Therefore, in the next experiment, we explored whether SJF alleviated inflammatory responses in CFAinduced arthritic rats.

RF has been reported to be implicated in the etiopathogenesis of arthritic, and it is a major biomarker associated with immune diseases, including rheumatoid arthritis. ${ }^{27} \mathrm{CRP}$ is another important inflammation biomarker in rheumatoid arthritis. $^{28,29}$ Arthritis is a systemic inflammatory disease and is characterized by excess pro-inflammatory enzymes, such as MMPs, and pro-inflammatory cytokines, including IL-6, IL-1 $\beta$, and TNF- $\alpha$, which resulted in joint inflammation, and cartilage damage. ${ }^{30}$ Previous reports have shown that the inflammatory process played an important role in the pathogenesis of arthritis. ${ }^{31}$ The relationship between bone homeostasis and inflammation has been attributed to the effects of pro-inflammatory factors such as IL- 6 , IFN- $\gamma$, TNF- $\alpha$, and IL-1 $\beta$ that are overexpressed in a rat model of arthritis and in arthritis patients. $^{32}$ It has been reported that a decrease in bone resorption and disease severity may be achieved by the inhibition of these proinflammatory cytokines. ${ }^{33}$ Besides, IL10 exerts anti-inflammatory effects and inhibits the progression of bone and cartilage pathology in arthritis. ${ }^{32}$ Therefore, the development of anti-inflammatory drugs is considered to be the main way to treat arthritis. ${ }^{34}$ Interestingly, our findings indicated that SJF decreased IL-6, PGE2, IFN- $\gamma$, TNF- $\alpha$, and IL-1 $\beta$ levels, as well as increased IL-10 level, indicating the anti-arthritis activity of SJF via the suppression of proinflammatory cytokines and the release of antiinflammatory cytokines. These results were in agreement with previous reports. SJF exerted anti-inflammatory effects via decreasing the production of pro-inflammatory cytokines and inflammation biomarkers in an atypical adenomatous hyperplasia rat model. ${ }^{35}$ 
MMPs are a class of enzymes involved in cartilage degradation targeting proteoglycans and type-II collagen and have been reportedly implicated in arthritis and osteoarthritis disease processes. ${ }^{36}$ In addition, as indicators of pathological states, MMPs are excellent biomarkers in the assessment and diagnosis of arthritis disease. ${ }^{37}$ It has been reported that MMP-1 plays a vital role in collagen degradation and the expression level of which is upregulated under arthritic conditions. ${ }^{38}$ MMP-13 possesses a strong ability to degrade CII, and other types of matrix molecules and collagens such as perlecan, and proteoglycan. ${ }^{39}$ Interestingly, in the present study, treatment with SJF (especially in highdoses) inhibited the MMP-1 and MMP-13 levels, indicating that the cartilage protecting role of SJF might be mediated via the inhibition of MMP-1 and MMP-13 levels.

On the other side, oxidative stress also contributed to the pathophysiology of arthritis. ${ }^{40}$ Reactive oxygen species such as superoxide and hydroxyl, as well as antioxidant systems including GPX, CAT, and SOD, exist in living organisms. Oxidative stress was induced when antioxidants level lower than reactive oxygen species. ${ }^{41}$ Moreover, the MAPK, NF-B signaling pathways were activated by excessive ROS, which further triggered inflammatory responses in cells of arthritis. ${ }^{8}$ The previous report has indicated that the activities of antioxidant enzymes like CAT, SOD, and GPx were lowered in patients with arthritis. ${ }^{42}$ Besides, MDA is a product of lipid peroxidation, which is reported to be higher in arthritis patients. ${ }^{43}$ In terms of our study, we observed that CFA evoked an obvious decline in SOD, CAT, and GPx activities and an increase in MDA content. These findings are in agreement with a previous report. ${ }^{15}$ And we found that SJF improved the activities of antioxidant enzymes (CAT, SOD, and GPx) and decreased the MDA content compared with CFA rats, indicating that SJF suppressed CFA-induced arthritis oxidation via its antioxidant action, thereby influencing infiltration and recruitment of neutrophils, and inhibiting the progression of arthritis.

In conclusion, for the first time, our findings indicated that SJF attenuated arthritis progression in CFA rats which could be partly attributed to inhibit inflammatory responses and attenuate oxidative stress. These results demonstrated that the therapeutic potential of SJF in the prevention and treatment of arthritis. However, this research also has some limitations. Although the beneficial effects of SJF were observed in a rat model of arthritis, the toxicity, maximum tolerated dose, and side effects of SJF in CFA rats should be further explored. In addition, the related clinical trial of SJF needs to be further investigated.

\section{Article Highlights}

1. SJ flower extract (SJF) attenuated arthritis symptoms in CFA rats.

2. SJF inhibited inflammatory responses and attenuated oxidative stress induced by CFA.

\section{Data Sharing Statement}

The data that support the findings of this study are available from the corresponding author, upon reasonable request.

\section{Funding}

The current work is supported by the fund of the Tianjin Science and Technology Commission Support Project (NO. 15ZXLCSY00040).

\section{Disclosure}

The authors declare that there are no conflicts of interest in this work.

\section{References}

1. Tang $\mathrm{CH}$. Research of pathogenesis and novel therapeutics in arthritis. Int J Mol Sci. 2019;20(7):1646. doi:10.3390/ijms20071646

2. Schett G, Gravallese E. Bone erosion in rheumatoid arthritis: mechanisms, diagnosis and treatment. Nat Rev Rheumatol. 2012;8 (11):656-664. doi:10.1038/nrrheum.2012.153

3. Li X, Wu Z, He B, Zhong W. Tetrandrine alleviates symptoms of rheumatoid arthritis in rats by regulating the expression of cyclooxygenase-2 and inflammatory factors. Exp Ther Med. 2018;16(3):2670-2676.

4. Aloke C, Ibiam UA, Obasi NA, et al. Effect of ethanol and aqueous extracts of seed pod of Copaifera salikounda (Heckel) on complete Freund's adjuvant-induced rheumatoid arthritis in rats. J Food Biochem. 2019;43(7):e12912. doi:10.1111/jfbc.12912

5. Direito R, Rocha J, Serra AT, et al. Anti-inflammatory effects of persimmon (Diospyros kaki L.) in experimental rodent rheumatoid arthritis. J Diet Suppl. 2019;1-21:663-683.

6. McInnes IB, Schett G. Cytokines in the pathogenesis of rheumatoid arthritis. Nat Rev Immunol. 2007;7(6):429-442. doi:10.1038/nri2094

7. McInnes IB, Schett G. The pathogenesis of rheumatoid arthritis. $N$ Engl $J$ Med. 2011;365(23):2205-2219. doi:10.1056/NEJMra1004965

8. Phull AR, Nasir B, Haq IU, Kim SJ, Umar S, Umar K. Oxidative stress, consequences and ROS mediated cellular signaling in rheumatoid arthritis. Chem Biol Interact. 2018;281:121-136. doi:10.1016/ j.cbi.2017.12.024

9. Yeom MJ, Lee HC, Kim GH, et al. Anti-arthritic effects of Ephedra sinica STAPF herb-acupuncture: inhibition of lipopolysaccharideinduced inflammation and adjuvant-induced polyarthritis. J Pharmacol Sci. 2006;100(1):41-50.

10. He X, Bai Y, Zhao Z, et al. Local and traditional uses, phytochemistry, and pharmacology of Sophora japonica L.: a review. J Ethnopharmacol. 2016;187:160-182. doi:10.1016/j.jep.2016.04.014

11. Liu J-L, Li L-Y, He G-H. Optimization of microwave-assisted extraction conditions for five major bioactive compounds from flos sophorae immaturus (Cultivars of Sophora japonica L.) using response surface methodology. Molecules. 2016;21(3):296. doi:10. 3390/molecules 21030296 
12. Li J, Gang D, Yu X, et al. Genistein: the potential for efficacy in rheumatoid arthritis. Clin Rheumatol. 2013;32(5):535-540. doi:10.1007/s10067-012-2148-4

13. Aa LX, Fei F, Qi Q, et al. Rebalancing of the gut flora and microbial metabolism is responsible for the anti-arthritis effect of kaempferol. Acta Pharmacol Sin. 2020;41(1):73-81. doi:10.1038/s41401-019-0279-8

14. Abd El-Ghffar EA, Eldahshan OA, Barakat A, Efferth T. The prophylactic effect of a Eugenia aquea extract against oxidative stress and inflammation associated with the development of arthritis in an adjuvant-induced arthritis rat model. Food Funct. 2018;9 (12):6643-6651. doi:10.1039/C8FO01570H

15. Zhang C, Zhang W, Shi R, Tang B, Xie S. Coix lachryma-jobi extract ameliorates inflammation and oxidative stress in a complete Freund's adjuvant-induced rheumatoid arthritis model. Pharm Biol. 2019;57 (1):792-798. doi:10.1080/13880209.2019.1687526

16. Fikry EM, Gad AM, Eid AH, Arab HH. Caffeic acid and ellagic acid ameliorate adjuvant-induced arthritis in rats via targeting inflammatory signals, chitinase-3-like protein-1 and angiogenesis. Biomed Pharmacother. 2019;110:878-886. doi:10.1016/j.biopha.2018.12.041

17. Wang T, Miao M, Bai M, et al. Effect of sophora japonica total flavonoids on pancreas, kidney tissue morphology of streptozotocin-induced diabetic mice model. Saudi J Biol Sci. 2017;24(3):741-747. doi:10.1016/j.sjbs.2017.01.051

18. Kuhad A, Chopra K. Tocotrienol attenuates oxidative-nitrosative stress and inflammatory cascade in experimental model of diabetic neuropathy. Neuropharmacology. 2009;57(4):456-462. doi:10.1016/j. neuropharm.2009.06.013

19. Ren S-X, Zhang B, Lin Y, Ma D-S, Li H. Mechanistic evaluation of antiarthritic activity of $\beta$-methylphenylalanine in experimental rats. Biomed Pharmacother. 2019;113:108730. doi:10.1016/j.biopha.2019.108730

20. Pan D, Li N, Liu Y, et al. Kaempferol inhibits the migration and invasion of rheumatoid arthritis fibroblast-like synoviocytes by blocking activation of the MAPK pathway. Int Immunopharmacol. 2018;55:174-182. doi:10.1016/j.intimp.2017.12.011

21. Gul A, Kunwar B, Mazhar M, et al. Rutin and rutin-conjugated gold nanoparticles ameliorate collagen-induced arthritis in rats through inhibition of NF- $\mathrm{KB}$ and iNOS activation. Int Immunopharmacol. 2018;59:310-317. doi:10.1016/j.intimp.2018.04.017

22. Haleagrahara N, Miranda-Hernandez S, Alim MA, Hayes L, Bird G, Ketheesan N. Therapeutic effect of quercetin in collagen-induced arthritis. Biomed Pharmacother. 2017;90:38-46. doi:10.1016/j. biopha.2017.03.026

23. Cheng WX, Huang H, Chen JH, et al. Genistein inhibits angiogenesis developed during rheumatoid arthritis through the IL-6/JAK2/STAT3/ VEGF signalling pathway. J Orthopaed Transl. 2020;22:92-100. doi:10.1016/j.jot.2019.07.007

24. Tsai SW, Lin CC, Lin SC, Wang SP, Yang DH. Isorhamnetin ameliorates inflammatory responses and articular cartilage damage in the rats of monosodium iodoacetate-induced osteoarthritis. Immunopharmacol Immunotoxicol. 2019;41(4):504-512. doi:10.1080/08923973.2019. 1641723

25. Park DS, Seo BK, Baek YH. Analgesic effect of electroacupuncture on inflammatory pain in collagen-induced arthritis rats: mediation by alpha2- and beta-adrenoceptors. Rheumatol Int. 2013;33(2):309-314. doi:10.1007/s00296-012-2369-5

26. Kumar A, Dhaliwal N, Dhaliwal J, Dharavath RN, Chopra K. Astaxanthin attenuates oxidative stress and inflammatory responses in complete Freund-adjuvant-induced arthritis in rats. Pharmacol Rep. 2020;72(1):104-114. doi:10.1007/s43440-019-00022-z

27. Ingegnoli F, Castelli R, Gualtierotti R. Rheumatoid factors: clinical applications. Dis Markers. 2013;35(6):727-734. doi:10.1155/2013/ 726598
28. Yang L, Liu R, Fan A, Zhao J, Zhang Y, He J. Chemical composition of pterospermum heterophyllum root and its anti-arthritis effect on adjuvant-induced arthritis in rats via modulation of inflammatory responses. Front Pharmacol. 2020;11:584849. doi:10.3389/ fphar.2020.584849

29. Pepys MB, Hirschfield GM. C-reactive protein: a critical update. J Clin Invest. 2003;111(12):1805-1812. doi:10.1172/ JCI200318921

30. Bingham CO. The pathogenesis of rheumatoid arthritis: pivotal cytokines involved in bone degradation and inflammation. $J$ Rheumatol. 2002;65:3-9.

31. McInnes IB, Schett G. Pathogenetic insights from the treatment of rheumatoid arthritis. Lancet (London, England). 2017;389 (10086):2328-2337. doi:10.1016/S0140-6736(17)31472-1

32. Juarranz Y, Abad C, Martinez C, et al. Protective effect of vasoactive intestinal peptide on bone destruction in the collagen-induced arthritis model of rheumatoid arthritis. Arthritis Res Ther. 2005;7(5):R1034R1045. doi:10.1186/ar1779

33. Umar S, Umar K, Sarwar AH, et al. Boswellia serrata extract attenuates inflammatory mediators and oxidative stress in collagen induced arthritis. Phytomed. 2014;21(6):847-856. doi:10.1016/j.phymed.20 14.02.001

34. Zhai KF, Duan H, Luo L, et al. Protective effects of paeonol on inflammatory response in IL-1 $\beta$-induced human fibroblast-like synoviocytes and rheumatoid arthritis progression via modulating NF- $\mathrm{kB}$ pathway. Inflammopharmacology. 2017;25:523-532. doi:10.1007/ s10787-017-0385-5

35. Elberry A, Mufti S, Al-Maghrabi J, et al. The protective effect of Sophora japonica on prostatic hypertrophy and inflammation in rat. Inflammopharmacology. 2020;28:1525-1536. doi:10.1007/s10787020-00723-5

36. Vincenti MP, Brinckerhoff CE. Transcriptional regulation of collagenase (MMP-1, MMP-13) genes in arthritis: integration of complex signaling pathways for the recruitment of gene-specific transcription factors. Arthritis Res. 2002;4(3):157-164. doi:10.1186/ $\operatorname{ar} 401$

37. Rose BJ, Kooyman DL. A tale of two joints: the role of matrix metalloproteases in cartilage biology. Dis Markers. 2016;20 16:4895050. doi:10.1155/2016/4895050

38. Wu H, Du J, Zheng Q. Expression of MMP-1 in cartilage and synovium of experimentally induced rabbit ACLT traumatic osteoarthritis: immunohistochemical study. Rheumatol Int. 2008;29 (1):31-36. doi:10.1007/s00296-008-0636-2

39. Shiomi T, Lemaître V, D’Armiento J, Okada Y. Matrix metalloproteinases, a disintegrin and metalloproteinases, and a disintegrin and metalloproteinases with thrombospondin motifs in non-neoplastic diseases. Pathol Int. 2010;60(7):477-496. doi:10.1111/j.14401827.2010.02547.x

40. García-González A, Gaxiola-Robles R, Zenteno-Savín T. Oxidative stress in patients with rheumatoid arthritis. Rev Invest Clin. 2015;67 (1):46-53.

41. Hitchon CA, El-Gabalawy HS. Oxidation in rheumatoid arthritis. Arthritis Res Ther. 2004;6(6):265-278. doi:10.1186/ar1447

42. Staroń A, Mąkosa G, Koter-Michalak M. Oxidative stress in erythrocytes from patients with rheumatoid arthritis. Rheumatol Int. 2012;32 (2):331-334. doi:10.1007/s00296-010-1611-2

43. Vijayakumar D, Suresh K, Manoharan S. Lipid peroxidation and antioxidant status in blood of rheumatoid arthritis patients. Indian J Clin Biochem. 2006;21(1):105. doi:10.1007/ BF02913075 


\section{Publish your work in this journal}

The Journal of Pain Research is an international, peer reviewed, open access, online journal that welcomes laboratory and clinical findings in the fields of pain research and the prevention and management of pain. Original research, reviews, symposium reports, hypothesis formation and commentaries are all considered for publication. The manuscript management system is completely online and includes a very quick and fair peer-review system, which is all easy to use. Visit http:// www.dovepress.com/testimonials.php to read real quotes from published authors.

Submit your manuscript here: https://www.dovepress.com/journal-of-pain-research-journal 